\title{
VARIABILIDAD GENÉTICA DE LA SHAPAJA Attalea moorei EN SEIS POBLACIONES NATURALES DE LA AM AZONÍA PERUANA
}

\author{
Diana CASTRO-RUIZ ${ }^{1}$, Ángel RODRÍGUEZ ${ }^{2}$, Werner CHOTA-MACUYAMA ${ }^{1}$, Dennis DEL- \\ CASTILLO $^{1}$, Víctor SOTERO ${ }^{2}$, Kember MEJIA ${ }^{1}$, Jean François RENNO ${ }^{3}$, Carmen GARCÍA-DÁ VILA ${ }^{1}$ \\ 1 Instituto de Investigaciones de la Amazonía Peruana (IIAP). A partado 784. Iquitos, Perú. E-mail: cdavila19@ yahoo.com \\ 2 Investigador Becado del proyecto IIAP-FINCYT. Iquitos \\ 3 Institut de Recherche pour le Développement-IRD. 175 Caviar, BP 5095, 34196 M ontpellier cedex 05, France.
}

\section{RESUMEN}

La diversidad genética poblacional de la Shapaja Athalea morei fue analizado mediante la técnica ISSR (Inter secuencias simples repetidas), en 6 localidades de la Amazonía Peruana, tres en el departamento de Loreto (Bagazán, Supay y Colpa) y tres en el departamento de San Martín (Cedamillo, Shapaja y Chayahuaqui). Se analizaron un total de 120 muestras (20 muestras de cada población) con dos marcadores ISSR: GACA y CAG. Los resultados del análisis factorial de Correspondencia (AFC), índice de fijación, distancia genética y el dendograma estimado por el método UPGMA muestran una fuerte diferenciación genética entre las poblaciones loretanas y las poblaciones Sanmartinenses. A nivel intrapoblacional, la población Chayahuaqui presentó mayor diversidad genética (6 genotipos) entre las 6 poblaciones estudiadas.

PALABRAS CLAVE: Athalea morei, Shapaja, diversidad genética, ISSR.

\section{GENETIC VARIABILITY OF SHAPAJ A Attalea moorei IN SIX NATURAL POPULATIONS OF THE PERUVIAN AM AZON}

\begin{abstract}
The population genetic variability in Shapaja Athalea morei was analized in six localities of the Peruvian Amazon, three in the Loreto region (Bagazán, Supay and Colpa) and three in the San Martin region (Cedamillo, Shapaja and Chayahuaqui). A total of 120 samples (20 samples from each population) were analyzed with the tecchnique ISSR "Inter simple sequence repeats" with two ISSR markers: GACA and CAG. The results of the AFC, index of fixation, genetic distance and dendrogram UPGMA estimated by the method show a strong genetic differentiation among populations of Loreto and San Martín. At Intrapopulation level, the population Chayahuaqui has the greatest genetic diversity (6 genotypes) of the six studied populations.
\end{abstract}

KEYW ORDS: Athalea morei, Shapaja, genetic diversity, ISSR 


\section{INTRODUCCIÓN}

A nivel mundial las palmeras incluyen 200 géneros y 2.450 especies distribuidas en la región tropical, con algunas especies que se extienden en áreas subtropicales en ambos hemisferios (Borchsenius \& Morães, 2006). Además de ser un grupo diverso y ecológicamente importante, la familia Arecacea proporciona diversos productos útiles y tienen un lugar importante en la vida cotidiana de la mayoría de las comunidades rurales (Wallace, 1853; Khan, 1991). Las palmeras, tienen un potencial económico como fruto comestible, aceite, palmito en conserva, fibras, almidón, construcción de viviendas, techado de casas, usos medicinales o constituyen un recurso que podría ser aprovechada para el mejoramiento genético de especies nativas cultivadas (Khan, 1991; Morães et al., 1996; Sosnowska \& Balslev, 2008). La Amazonía peruana posee en su diversidad biológica un gran número de palmeras que podrían ser utilizadas para el desarrollo de diversos productos alimenticios, medicinales e industriales. Dentro de ellas tenemos al género Attalea, cuyas especies se presentan tanto como pequeñas palmeras o como plantas de gran tamaño. Este género está distribuido en toda la región Neotropical desde México hasta Bolivia, Paraguay, Sur de Brasil y en algunas islas Caribeñas, encontrándose desde las dunas de arena costeras hasta el bosque sub-Andino, atravesando por todo tipo de bosque tropical, seco o húmedo, pantanos y sabanas (Pintaud, 2008). Los frutos de estas especies son utilizadas en la medicina tradicional con fines antiinflamatorios, antirreumáticos, para el tratamiento de la leucemia, contra la obesidad, como anticonceptiva, antitrombótico; además de poseer un endospermo rico en ácidos grasos esenciales, el cual les convierte en especies de elevado potencial económico (Albán et al., 2008)

La identificación de estas especies según Pintaud (2008) es poco entendida por los conflictos de conceptos entre los taxonomistas y por la carencia de muestras en los herbarios especialmente de las especies de gran tamaño. En este sentido los marcadores moleculares son herramientas indispensables para dilucidar problemas de dudosa identificación, estableciendo límites confiables, así como también para evaluar la diversidad genética. El desarrollo de los marcadores moleculares basados en DNA ha causado un fuerte impacto en la genética vegetal. Ya que permiten evaluar la variabilidad genética inter e intra poblacional, estimar las distancias genéticas entre poblaciones, identificar variedades, líneas puras, establecer relaciones de parentesco y acelerar los programas de mejoramiento genético (Ferreira \& Grattapaglia, 1998). Así mismo la estimación de la variabilidad genética con técnicas moleculares es muy usada porque permite conocer el grado de conservación de las especies para establecer futuros programas de manejo sostenido. En el caso de las palmeras del género Attalea, fueron utilizados marcadores ISSR, debido a que hasta el momento no existen marcadores específicos para este grupo. Los marcadores ISSR (inter secuencias simples repetidas) son marcadores universales (que pueden ser utilizados en un grupo grande de individuos) que se caracterizan por generan una buena información sobre la diferenciación genética entre organismos cercanamente relacionados sin necesidad de información previa de la secuencia del genoma a investigar (Bornet \& Branchard, 2001; Rodriguéz et al., 2010; Djè et al., 2006; Bornet et al., 2002a; Bornet et al., 2002b; Bornet \& Branchard, 2001; Zietkiewicz, et al., 1994).

Este estudio mediante los ISSR, pretende evaluar el nivel de variabilidad genética intraespecifica de Attalea moorei, con la finalidad de determinar el grado de variabilidad genética en las poblaciones naturales, así como las relaciones entre ellas. Los resultados generados servirán de base para la elaboración de futuros planes de manejo sostenido para la especie.

\section{ATERIALES Y MÉTODOS}

\section{COLECTA DE MATERIAL BIOLÓGICO Y EXTRACCIÓN DE ADN}

La variabilidad genética de Attalea moorei fue evaluada en base de 20 plantas por cada seis poblaciones naturales: Loreto (Bagazán, Supay y Colpa), San Martín (Cedamillo, Shapaja y Chayahuaqui), (Figura 1).

La extracción de ADN, fue realizada mediante el protocolo CTAB de Doyle \& Doyle (1987), a partir de $100 \mathrm{mg}$ de tejido foliar. La amplificación del ADN se realizó con los primers GACA (GACAGACAGACAGACA) y CAG (CAGCAGCAGCAGCAG), diseñados por Bornet y Branchard (2001); de acuerdo a la técnica Inter Simple Sequence Repeat-ISSR (Zietkiewicz et al., 1994). La reacción de amplificación fue realizada en un volumen total de 25 $\mu \mathrm{l}$, conteniendo $5 \mathrm{U} / \mu \mathrm{l}$ de Taq polimerasa, $100 \mathrm{ng} / \mu \mathrm{l}$ de ADN molde, 5X de Buffer, $10 \mathrm{mM}$ dNTPs, $25 \mathrm{mM}$ de $\mathrm{MgCl} 2,10 \mu \mathrm{M}$ de cada primer y agua ultrapura. Las condiciones de temperatura fueron: denaturación inicial a $95{ }^{\circ} \mathrm{C}$ x $1 \mathrm{~min}$; seguida de 35 ciclos de: denaturación a $94^{\circ} \mathrm{C}$ x $30 \mathrm{~s}$, hibridación a $59.5^{\circ} \mathrm{C}$ x 30 $\mathrm{s}$, y extensión a $72{ }^{\circ} \mathrm{C} \times 1 \mathrm{~min}$; seguida de una extensión final a $72{ }^{\circ} \mathrm{C}$ x 10 min. El patrón de bandas obtenido, fue verificado en geles de poliacrilamida al $6 \%$, teñido con nitrato de plata mediante el método Rabat (Sambrook \& Russell, 1991). 


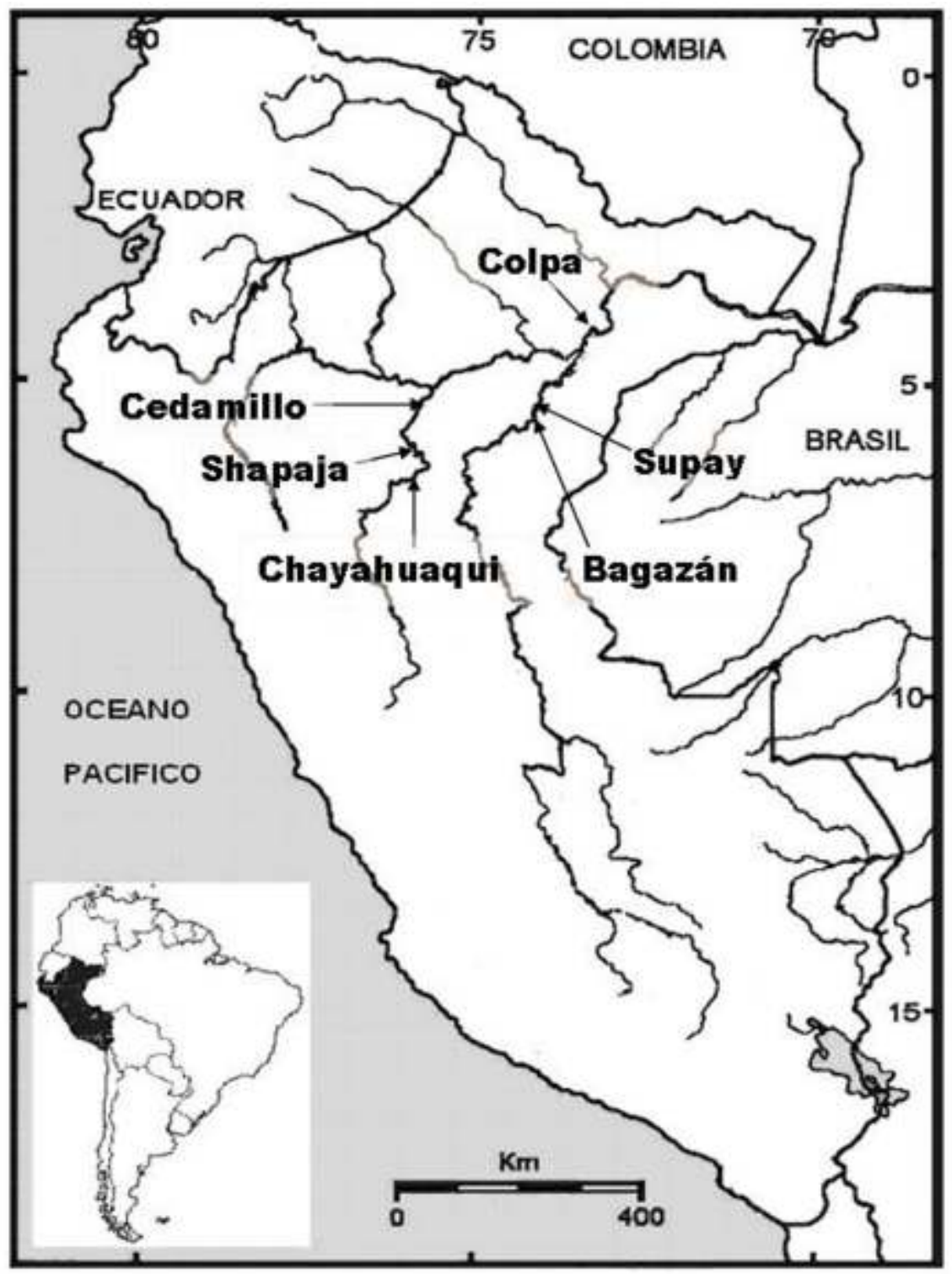

Figura 1. Mapa mostrando la ubicación geográfica de las seis poblaciones naturales de Attalea moorei estudiadas.

\section{ANÁLISIS DEDATOS}

El polimorfismo entre las poblaciones fue analizado mediante la observación de presencia y ausencia de las bandas entre los individuos con los primers GACA y CAG. La variabilidad genética fue establecida mediante el Análisis Factorial de Correspondencia (AFC) considerante el genotipo individual. La diferenciación entre las poblaciones fue estimada en base al índice de fijación $\left(\mathrm{F}_{\mathrm{ST}}\right)$ propuesto por Weir \&
Cockerham (1984), y las relaciones entre las agrupaciones fueron establecidas en base a un dendrograma (método UPGMA), elaborado a partir de la distancia genética (D) obtenida según Reynolds et al. (1983). Los análisis fueron realizados con la ayuda de los Softwares GENETIX versión 4.05 (Belkhir et al., 2004), PHYLIP versión 3.5 (Felsenstein, 1993) y TREEVIEW (Page, 1996). 


\section{RESULTADOS Y DISCUSIONES}

\section{RELACIONES FILOGEOGRÁFICAS DE Attalea moorei}

Las poblaciones naturales de la shapaja presentaron diferentes niveles de variabilidad genética poblacional. Se obtuvieron 12 bandas informativas (bandas diferenciales o polimorficas) con los dos marcadores ISSR analizados: GACA con 9 bandas informativas $(75 \%)$ y CAG con tres bandas informativas (25\%). Asimismo fueron encontrados 22 perfiles genéticos (genotipos) entre los 120 individuos de las seis poblaciones estudiadas, siendo que las poblaciones sanmartinenses presentaron mayor diversidad genética (14 genotipos) que las poblaciones de Loreto (8 genotipos). Se observó que Chayahuaqui fue la población con mayor diversidad intrapoblacional (6 genotipos), así como también Bagazán y Colpa son poblaciones que comparten dos genotipos entre sí (Tabla 1).

Los resultados del Análisis Factorial de Correspondencia, AFC (Figura 2), muestran una clara diferenciación entre las poblaciones loretanas (Supay, Colpa y Bagazán) y las poblaciones sanmartinenses (Cedamillo, Shapaja y Chayahuaqui), observándose dos grupos diferenciados sin sobreposición entre ellos. La estructura que muestra la AFC esta probada por la distribución por pares del índice de fijación $\left(\mathrm{F}_{\mathrm{st}}\right)$ utilizado para establecer la distancia genética (Tablas 2 y 3),. Siendo que los mayores índices son presentados por Colpa y Shapaja $\left(\mathrm{D}=1.8368 ; \mathrm{F}_{\mathrm{st}}\right.$ $=0.8407)$ y los menores los encontrados entre Colpa $\mathrm{y}$
Bagazán $\left(\mathrm{D}=0.0962 ; \mathrm{F}_{\mathrm{st}}=0.0917\right) . \quad \mathrm{El}$ dendograma obtenido mediante el método UPGMA (Figura 3) muestra que las poblaciones forman dos principales agrupaciones: la primera conformada por las poblaciones de San Martín (Cedamillo, Shapaja y Chayahuaqui), dentro del cual se observa una subagrupación constituida por Shapaja y Chayahuaqui. La segunda agrupación lo conforman las poblaciones de Loreto (Bagazán, Colpa y Supay), siendo que Colpa y Bagazán son las más cercanamente relacionadas. La cercana relación entre las poblaciones sanmartinenses como entre las poblaciones loretanas de shapaja nos sugiere que esta palmera en el pasado quizás constituía amplios manchales en la Amazonía. Posteriormente por ocupación antropogénica del área, los manchales fueron sufriendo un constante fraccionamiento, con el aislamiento de algunas de sus poblaciones lo que conllevo a una fuerte erosión genética en algunas de ellas (Por ejemplo Bagazán y Colpa).

También existen estudios donde utilizaron marcadores DALP para evaluar la distancia genética en tres poblaciones naturales de otras especies de palmeras como Mauritia flexuosa, (Aspajo et al., 2008). Los resultados mostraron que las tres poblaciones se encuentran bien diferenciadas, Esta diferenciación es atribuido a las grandes distancias geográficas que combinadas a la existencia de los ríos entre las poblaciones podrían estar actuando como barreras naturales que restringen el flujo genético. 
Tabla 1. Genotipos encontrados en las seis poblaciones de Attalea moorei con dos marcadores ISSR.

\begin{tabular}{|c|c|c|c|c|c|}
\hline \multirow[b]{2}{*}{ POBLACIÓN } & \multirow{2}{*}{$\begin{array}{l}\text { NOM BRE DE } \\
\text { GENOTIPO }\end{array}$} & \multirow{2}{*}{$\begin{array}{l}\text { NÚMERO DE } \\
\text { INDIVIDUOS } \\
\text { POR GENOTIPO }\end{array}$} & \multicolumn{2}{|l|}{ GENOTIPOS } & \multirow[b]{2}{*}{ GENOTIPO } \\
\hline & & & $\begin{array}{l}\text { MARCADOR } \\
\text { GACA }\end{array}$ & $\begin{array}{l}\text { MARCADOR } \\
\text { GAC }\end{array}$ & \\
\hline \multirow{3}{*}{ Bagazán } & $\mathrm{Bg} 1$ & 4 & $B A B B A A A B$ & B B A & $B A B B A A A B B A$ \\
\hline & Bg2 & 10 & B B B A B A A A B & B B A & B B B A B A A B B B A \\
\hline & * Bg3 & 5 & $B B$ B B BA B & $B$ B A & $B B B B B A B B A$ \\
\hline \multirow{4}{*}{ Supay } & Su1 & 9 & $B B B A B A A B$ & $B B A$ & $B B B A B A B B B B A$ \\
\hline & Su2 & 2 & $B B B A B B A B$ & $B B A$ & $B B B A B B A B B A$ \\
\hline & Su3 & 6 & $B B B B B B A B$ & $B B A$ & $B B B B B B A B B A$ \\
\hline & Su4 & 3 & $B$ B B A B A A A B & $\mathrm{B} \mathrm{B} \mathrm{A}$ & $B B B A B A A A B B A$ \\
\hline \multirow{3}{*}{ Colpa } & $\mathrm{Col}$ & 6 & $B B B A B A A B$ & $B B A$ & $B B B A B B A B B B A$ \\
\hline & $\mathrm{Co2}$ & 8 & В В В А В А А А & B B A & B B B A B A A B B B A \\
\hline & * Co5 & 6 & $B D B D B A$ & B B A & $B D B B A A B D A$ \\
\hline \multirow{4}{*}{ Cedamillo } & $\mathrm{Cd} 1$ & 8 & $B A A B A B A A$ & $A B B$ & $B A A B B A A A A B B$ \\
\hline & $\mathrm{Cd} 2$ & 6 & $B A A B B B B A$ & $A B B$ & $B A A B B B B A B B$ \\
\hline & $\mathrm{Cd} 3$ & 2 & $B B A B B A A A$ & $A B B$ & $B B A B B A A A A B B$ \\
\hline & $\mathrm{Cd} 4$ & 4 & $B A A B B A B B$ & $A B B$ & $B A A B B A B B A B B$ \\
\hline \multirow{4}{*}{ Shapaja } & Sh1 & 7 & $B A A B A B B B$ & $A A B$ & $B A A B A B B B A A B$ \\
\hline & Sh2 & 9 & $B A A B B B B$ & $A A B$ & $B A A B B B B B A B$ \\
\hline & Sh3 & 2 & $A A A B A B A A$ & $A A B$ & $A A A B A B A A A B$ \\
\hline & Sh4 & 2 & $A A A B A B A B$ & $A A B$ & $A A A B A B A B A A B$ \\
\hline \multirow{6}{*}{ Chayahuaqui } & Ch1 & 2 & $A A A B A B A B$ & $A B B$ & $A A A B A B A B A B$ \\
\hline & $\mathrm{Ch} 2$ & 2 & $A A A B A B A B$ & $A B B$ & $A A A B B A A B A B$ \\
\hline & Ch3 & 2 & $A A A B A B B B$ & $A B B$ & $A A A B B A B B A B B$ \\
\hline & Ch4 & 6 & $B A A B B B B B$ & $A B B$ & $B A A B B B B B A B B$ \\
\hline & Ch5 & 5 & $A A A B B B A B$ & $A B B$ & $A A A B A B A B A B B$ \\
\hline & Ch6 & 3 & $A A A B B B A B$ & $A B B$ & $A A A B B B A B A B B$ \\
\hline
\end{tabular}

* Genotipo compartido 


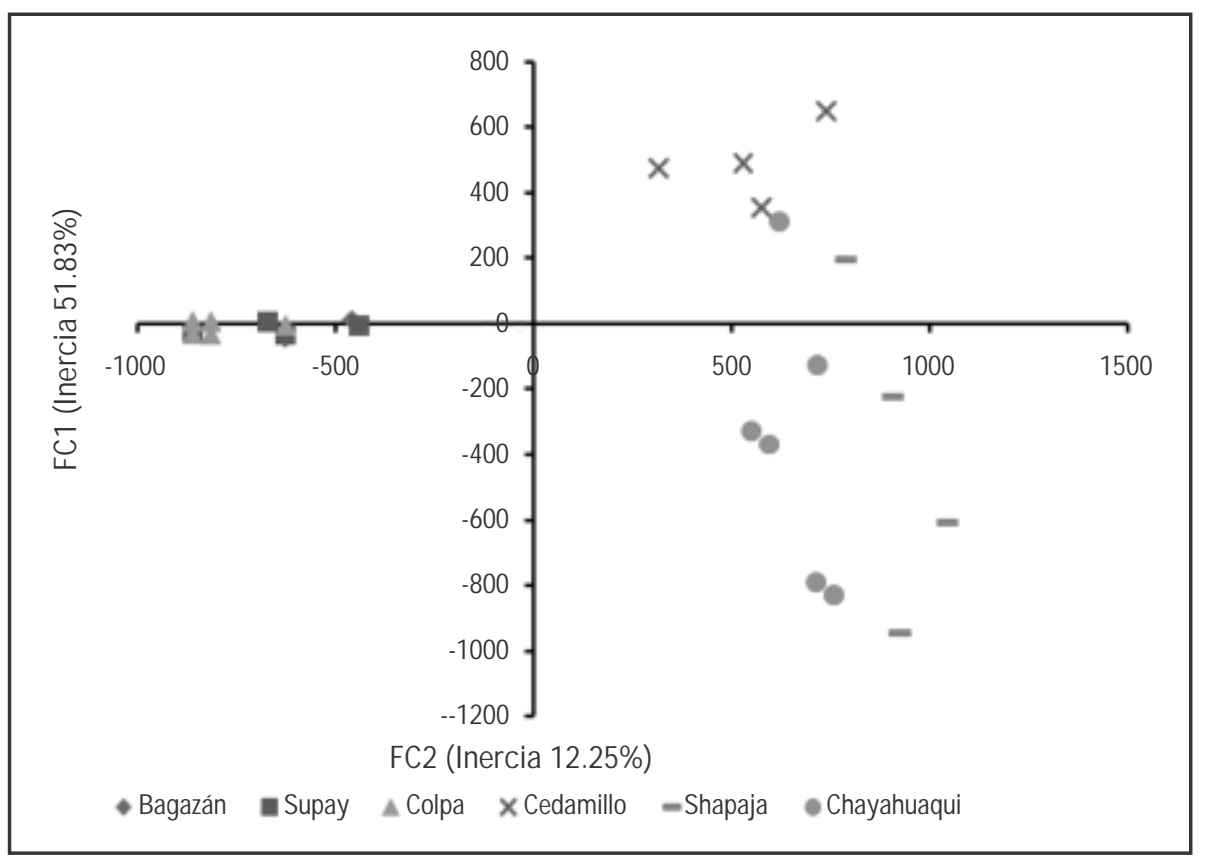

Figura 2. Representación gráfica de los resultados del Análisis Factorial de Correspondencia (AFC). Los puntos están distribuidos sobre un plano bidimensional: eje 1 (inercia 51.83\%). eje 2 (inercia 12.25\%) para las seis poblaciones naturales de Attalea moorei.

Tabla 2. M atriz del estimador $\mathrm{F}_{\mathrm{st}}$ de Weir \& Cockerham, 1984 para las seis poblaciones naturales analizadas de Attalea moorei de la Amazonía peruana.

\begin{tabular}{ccccccc}
\hline POBLACIÓN & BAGAZÁN & SUPAY & COLPA & CEDAMILLO & SHAPAJ A & CHAYAHUAQUI \\
\hline Bagazán & 0.000 & 0.373 & 0.092 & 0.799 & 0.814 & 0.784 \\
Supay & - & 0.000 & 0.396 & 0.792 & 0.808 & 0.775 \\
Colpa & - & - & 0.000 & 0.836 & 0.841 & 0.815 \\
Cedamillo & - & - & - & 0.000 & 0.540 & 0.445 \\
Shapaja & - & - & - & - & 0.000 & 0.423 \\
Chayahuaqui & - & - & - & - & - & 0.000 \\
\hline
\end{tabular}

*** Altamente significativo a $\mathrm{P}<0.001$ 
Tabla 3. Distancia genética de Reynolds, W eir \& Cockerham, 1983 para las seis poblaciones naturales de Attalea moorei.

\begin{tabular}{ccccccc}
\hline POBlACIÓN & BAGAZÁN & SUPAY & COLPA & CEDAMILLO & SHAPAJ A & CHAYAHUAQUI \\
\hline Bagazán & 0.000 & 0.4666 & $\mathbf{0 . 0 9 6 2}$ & 1.6037 & 1.6812 & 1.5302 \\
Supay & - & 0.0000 & 0.5038 & 1.5693 & 1.6517 & 1.4896 \\
Colpa & - & - & 0.0000 & 1.8072 & 1.8368 & 1.6874 \\
Cedamillo & - & - & - & 0.0000 & 0.7766 & 0.5888 \\
Shapaja & - & - & - & - & 0.0000 & 0.5503 \\
Chayahuaqui & - & - & - & - & - & 0.0000 \\
\hline
\end{tabular}

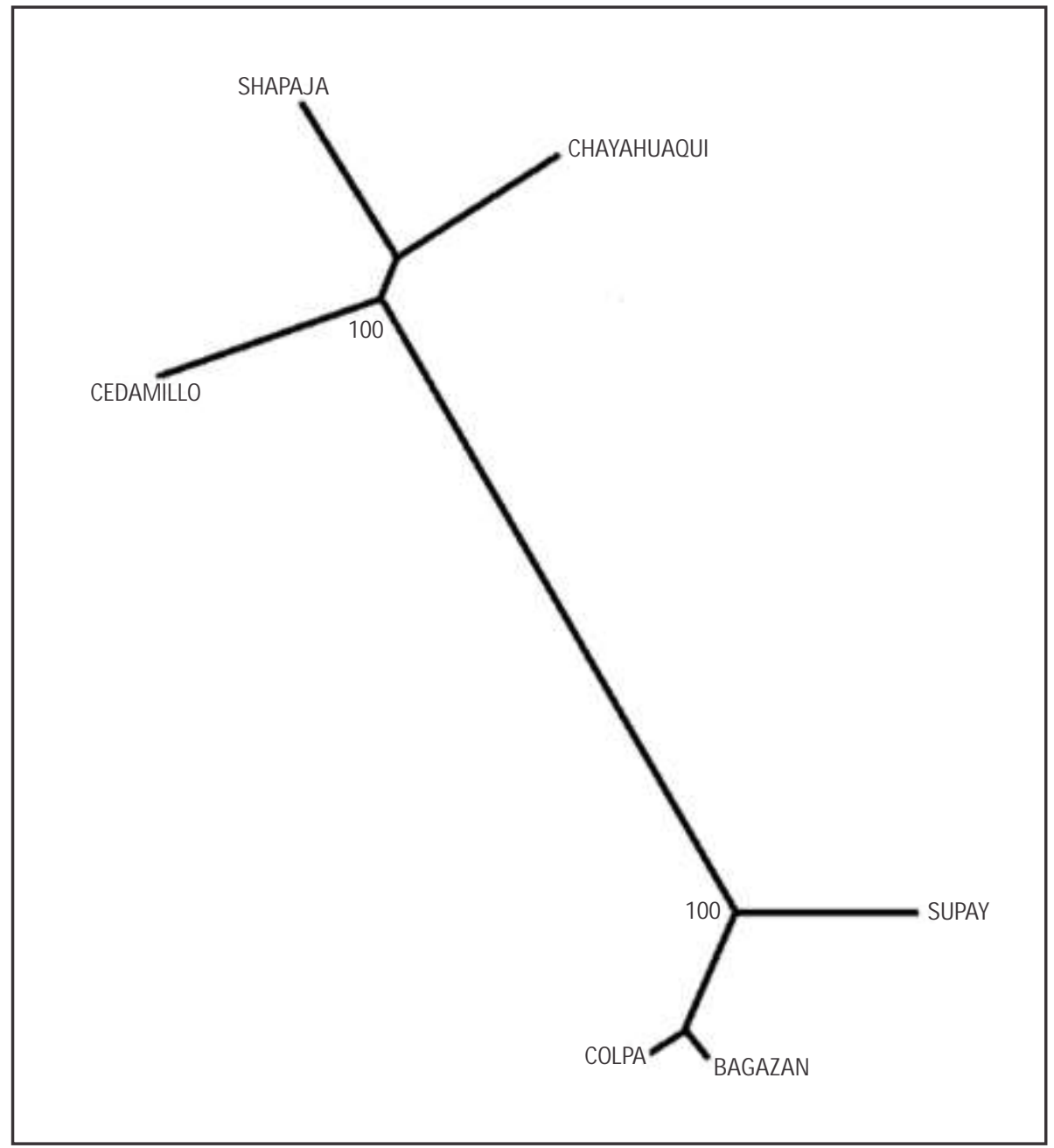

Figura 3. Dendograma UPGM A generado a partir de las distancias genéticas según Reynolds (1983) obtenidas entre seis poblaciones naturales de Attalea moorei. 


\section{AGRADECIMIENTO}

Al programa de ciencia y tecnología - FINCyT, por el financiamiento parcial del presente estudio a través del proyecto: "Potencial Nutracéutico, Caracterización química y genética de palmeras promisorias del complejo Attalea: Attalea moorei (shapaja), Attalea sp. (shebón), Attalea salazarii (sheboncillo)".

\section{REFERENCIAS BIBLIOGRAFICAS}

Albán, J.; Millán, B.; Khan, F.2008. Situación actual de la investigación etnobotánica sobre palmeras de Perú. Revista Peruana de Biología, 15(1): 133142.

Aspajo, F.; Rodríguez, A.; Corazón-Guivin, M.; Freitas, L.; Del Castillo, D.; Castro, D.; García, M.; Renno, J.; García-Dávila, C. 2008. Caracterización Genética de los Morfotipos de Aguaje Mauritia flexuosa L.F. (arecaceae) y análisis de la variabilidad de tres poblaciones naturales en la Amazonía peruana. Folia Amazónica. V 17(1-2): 75-82.

Belkhir, K.; Borsa, P.; Chichi, I.; Raufast, N. \& Bonhomme, F. 2004. GENETIX 4.05.2, logiciel sous windowns TM pour la genétique des populations. Laboratoire génome, populations, interactions, CNRS UMR 5000, Université de Montpellier II, Montpellier, France.

Borchsenius, F.; Moraes, M. 2006. Diversidad y usos de palmeras andinas (Arecaceae). Botánica Económica de los Andes Centrales, 412-433.

Bornet, B.; Branchard, M. 2001. Nonanchored intersimple sequence repeat (ISSR) markers: reproducible and specifics tools for genome fingerprinting. Plant Molecular Biology Reporter, 19:209-215

Bornet, B.; Muller, C.; Paulus, F.; Branchard, M. 2002a. Highly informative nature of inter simple sequence repeat (ISSR) sequences amplified using tri- and tetra-nucleotide primers from DNA of cauliflower (Brassica oleracea var. botrytis L.). Genome, 45: 890-896.

Bornet, B.; Goraguer, F.; Joly, G.; Branchard, M. 2002b. Genetic diversity in European and Argentinian cultivated potatoes (Solanum tuberosum subsp. tuberosum) detected by intersimple sequence repeats (ISSRs). Genome, 45: 481-484.

Djè, Y.; Tahi, G.C.; Zoro, B. A.; Malice, M.; Baudoin, J.P.; Bertin, P. 2006. Optimization of ISSR marker for African edible-seeded Cucurbitaceae species genetic diversity analysis. African Journal of Biotechnology, 5(2): 83-87.
Doyle, J. J.; Doyle J. L. 1987. A rapid DNA isolation procedure for small quantities of fresh leaf tissue. Phytochem. Bull., 19:11-15.

Felsenstein, J. 1993. PHYLIP (Phylogeny Inference Package), version 3.5 c. Seattle: University of Washington.

Ferreira, M.Y.; Grattapaglia, D. 1998. Introducción al uso de marcadores moleculares en el análisis genético. EMBRAPA-CENARGEN, Brasilia, Brasil. 220pp.

Kahn, F.199 1. Palms as key swamp forest resources in Amazonia. Forest. Ecology and Management, 38: 133-142.

Moraes, M.; Borchsenius, F.; Blicher-Mathiesen, U.1996. Notes on the Biology and uses of the motacij palm (Athalea phalerata, Arecaceae) from Bolivia.Economic Botany, 50 (4): 423-428.

Page, R.D.M., 1996. Tree View v.1.40. http://taxonomy.zoology,gla.ac.uk/rod/rod.html

Pintaud, J.C. 2008. An overview of the taxonomy of Attalea (Arecaceae). Revista Peruana de Biología, 15 (1): 55-63.

Reynolds, J.; Weir, B. S.; Cockerham, C. C. 1983. Estimation of coancestry coefficient: basis for a short-term genetic distance. Genetics, 105: 767779.

Rodriguez, A,; Corazón-Guivin, M.; Cachique, D.; Mejía, K.; Del Castillo, D.; Renno, J.F.; GarcíaDávila, C. 2010. Diferenciación morfológica y por ISSR (Inter simple sequence repeats) de especies del género Plukenetia (Euphorbiaceae) de la Amazonía peruana: propuesta de una nueva especie. Revista Peruana de Biología, 17(3): 325330.

Sambrook, J.; Russell, D. 1991. Molecular Cloning: A laboratory Manual, 3rd ed. New York: Cold Spring Harbor Laboratory Press. 999pp.

Sosnowska, J,; Balslev, H. 2008. American palms used for medicine, in the ethnobotanical and pharmacological publications. Revista peruana de Biología, 15(1): 143-146.

Wallace, A.R. 1853. Palm Trees of the Amazon and their Uses. John Van Voorst, London. 243pp.

Weir, B.S., Cockerham, C. 1984. Estimating Fstatistics for the analysis of population structure. In: Evolution, Vol. 38, 1358-1370p.

Zietkiewicz, E,; Rafalski, A.; Labuda, D. 1994. Genome fingerprinting by simple sequence repeat (SSR)-anchored polymerase chain reaction amplification. Genomics 20: 176-183. 